\title{
Rhythms of the heart: The history of electrophysiology and its current status in South Africa
}

\author{
Rob Scott Millar* and Ashley Chin* \\ *Department of Medicine, Division of Cardiology, Groote Schuur \\ Hospital, University of Cape Town, Observatory, South Africa \\ \#Cardiac Clinic, Groote Schuur Hospital, University of Cape Town, \\ Observatory, South Africa
}

\section{Address for correspondence:}

Emeritus Associate Professor R Scott Millar

Department of Medicine

Division of Cardiology

E25, Groote Schuur Hospital

University of Cape Town

Main Road

Observatory

Cape Town

7925

South Africa

\section{Email:}

rob.scottmillar@gmail.com

\section{INTRODUCTION}

The field of cardiac electrophysiology (EP) has progressed from a niche activity practised by a few enthusiasts to one of the most popular branches of cardiology, thanks to the expanding therapeutic options offered by catheter ablation of arrhythmias and the increasing indications for cardiac implantable electronic devices (CIEDs). This review will look at the origins of the study of electrical phenomena in the heart and the growth of that knowledge. The status of EP in South Africa and sub-Saharan Africa will be discussed.

\section{I9TH CENTURY - ANATOMY AND PHYSIOLOGY}

While there are references to arrhythmias in ancient times and Claudius Galen in the second century AD observed that a heart which had been excised and isolated, continued to beat on its own, (1) most of the important work on the basic anatomy and physiology of the electrical system of the heart took place in the 19 th and early 20th centuries. This work formed the foundation for understanding cardiac electrophysiology and arrhythmias.

In 1839 Jan Evangelista Purkinje described the fibres bearing his name from observations made in sheep hearts. (2) He did not, however, have any idea as to their true function. Wilhelm His Jr

\section{ABSTRACT}

The foundations of cardiac electrophysiology were laid in the 19th and 20th century by the elucidation of the anatomy and physiology of the conduction system and cardiac muscle. The development of the ECG by Einthoven in the early 1900s has been the basis for its clinical application and remains the most important tool for diagnosis of arrhythmias. Invasive electrophysiological studies have refined and added to this understanding and led to the development of catheter ablation of cardiac arrhythmias. The invention of pacemakers and implantable cardioverter defibrillators (ICDs) has revolutionised the treatment of patients with brady and tachyarrhythmias. Despite these advances, catheter ablation, pacemakers and ICDs are unavailable to the majority of patients living in sub-Saharan Africa. In South Africa, there is a paucity of electrophysiologists in the academic/public sector as almost all electrophysiologists serve patients with private health insurance. SAHeart 2015;12:156-163

in 1883 described the penetrating bundle of His, but did not prove that it conducted the electrical impulses from the atria to the ventricles. ${ }^{(2)}$

During the 19th century, there were two theories as to the origin of cardiac rhythmicity. One view held that it was secondary to nervous stimulation via ganglia which had been observed on the heart. The other was that it was an intrinsic property of cardiac muscle. A key finding by Walter Gaskell in the 1880s was that an isolated strip of tortoise ventricular muscle, devoid of ganglia or nerves, continued to contract rhythmically at a rate similar to the intact heart, proving its intrinsic rhythmicity and he was responsible for elucidating the effects of the autonomic nervous system on the heart. Gaskell also followed up on the original experiments by Stannius in 1852 and Luciani in 1873, in which a ligature around the AV groove resulted in AV block. . $^{(1,23)}$

It was Sunao Tawara from Japan, working in the laboratory of Ludwig Aschoff in Marburg, Germany, who discovered the AV node and delineated the continuity of the conduction system from the AV node, via the His bundle and bundle branches, to the ventricular myocardium. He published his findings in a beautifully illustrated monograph in 1906. There was now a sound anatomical and physiological basis for AV conduction. ${ }^{(4)}$ 
The picture was completed by the discovery of the sinus node in 1907 by Arthur Keith and Martin Flack. (1.5) This was subsequently confirmed to be the site of origin of the cardiac impulse by Thomas Lewis, using Einthoven's electrocardiogram on the exposed heart.(2)

Karel Wenckebach in 1904 described the form of AV block that bears his name by means of pulse recordings, before the ECG became available. He was able to explain the original observations of this phenomenon made by Luciani in $1873 .{ }^{(6)}$

\section{EARLY 20TH CENTURY}

\section{Development of the ECG}

While Augustus Waller was the first to record a recognisable ECG in 1887,(7) the capillary electrometer which he used produced very low fidelity recordings. It was Willem Einthoven's modification of the string galvanometer which enabled successful recording of the heart's electrical activity in the first few years of the 20th century. ${ }^{(8)}$ His use of bipolar limb leads and

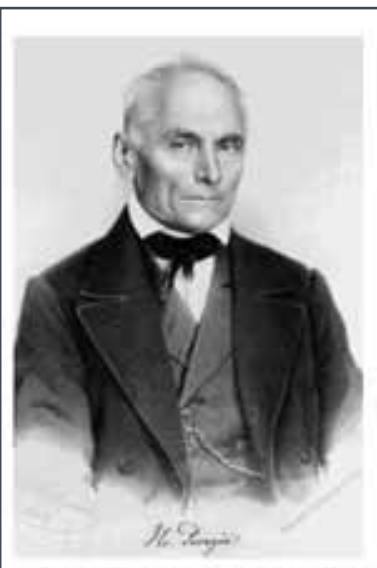

Johann Evangelist Purkinje: 1787-1869

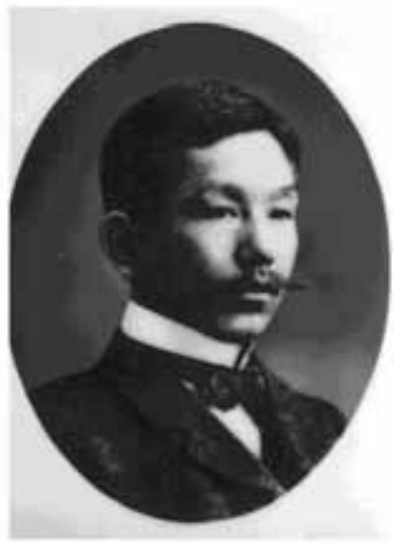

Suneo Tawara: $1873-1952$

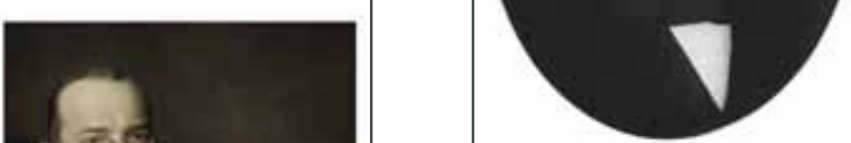

Willem Einthoven: 1860-1927

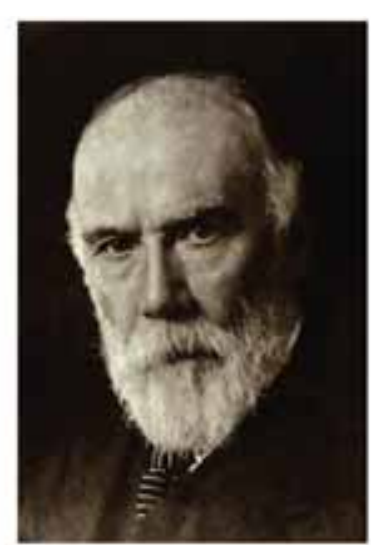

James Mackenzie: 1853-1925

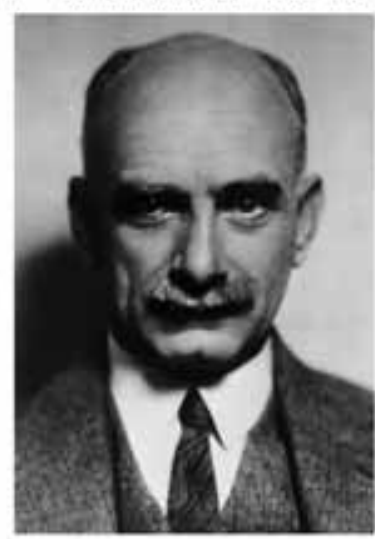

Thomas Lewis: 1881-1945
FIGURE 2: Gaskell proved the myogenic origin of the cardiac impulse and investigated AV block and autonomic influences on the heart. Mackenzie and Lewis clarified atrial fibrillation, together with other insights. Einthoven developed the ECG as a practical tool and Lewis established its place in clinical practice.

the names he gave to the waves survive to this day. While Einthoven recognised the value of the ECG, it was Sir Thomas Lewis in London who pioneered its use as a clinical tool.

William Hofmeyer (Don) Craib, a young South African on a Rockefeller fellowship to Harvard, challenged the prevailing negativity theory of Einthoven and Lewis. This described negativity as the major change at the surface of excited cardiac tissue. Craib conducted experiments which showed that this was not the case. He found that there were polyphasic swings in potential, from positive to negative and back again, during the passage of an excitation wave. He ascribed the differences between his findings and those of Lewis to his closer simulation of the heart by surrounding the muscle in saline, versus Lewis' thin film of saline. ${ }^{(9)}$ He then proposed his doublet hypothesis in 1927, causing much controversy. He described an excitation wave as a succession of "doublets". A "doublet" comprised of a simultaneous anode and cathode where current
FIGURE I: Purkinje, His and Tawara defined the AV conducting system. Keith, together with Flack, discovered the sinus node.

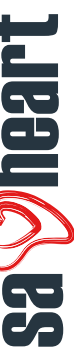


entered and exited a small fraction of muscle. The electrical activation proceeded in wave fronts from negative to positive. The idea was subsequently adopted and modified by Wilson as the dipole theory, which is still accepted today as the basis for the ECG.

The ECG has played a critical role in the understanding of cardiac arrhythmias and continues to be an indispensable tool in diagnosis and prognosis to this day. While ECG recording has been modified since Einthoven's day to include the unipolar limb leads and chest leads by Wilson and recording techniques have been refined by new technology, the fundamentals of the ECG have not changed in more than 100 years. Because of this long history, interpretation has been progressively refined as new knowledge becomes available. As a result, it remains a uniquely valuable clinical tool in all aspects of cardiology, particularly in the diagnosis of cardiac arrhythmias.

Atrial fibrillation (AF) was the first arrhythmia to attract major interest and research. The condition had long been known under a variety of names: "rebellious palpitations", "delirium cordis" and "pulsus irregularis perpetuus". James Mackenzie, a Scottish general practitioner from 1879 in Burnley, England, devoted much of his life to studying heart disease, and is considered one of the fathers of modern cardiology. ${ }^{(10)}$ Using an ink-writing polygraph which he had helped develop, he recorded simultaneous jugular and carotid pulses. He described the $a, c$ and $v$ waves of the jugular pulse, and noted the disappearance of the a waves with the development of an irregular pulse in a patient with mitral stenosis. His interpretation of atrial standstill and "nodal rhythm" was corrected by Thomas Lewis in 1909 as a result of his recording of fibrillary waves on the ECG, using Einthoven's string galvanometer. A little earlier, in 1909, Rothberger and Winterberg in Vienna had demonstrated fibrillation in experimental preparations. Einthoven had published an ECG in 1906 which clearly demonstrated $A F$, but he did not recognise its significance.

The concept of re-entry

In 1913 George Mines, a young man in his 20s, did experiments on the electrical activity of circular strips of muscle from a turtle heart. He was able to demonstrate the phenomenon of re-entry and describe the conditions of refractoriness and conduction which made it possible. (II) His prescient comment: "I venture to suggest that a circulating excitation of this type may be responsible for some cases of paroxysmal tachycardia as observed clinically", proved to be something of an understatement. Sadly, he died before the age of 30 and never lived to see this prediction come true.

\section{MID-20TH CENTURY}

\section{Refinement of the ECG}

In the 1930s and 40s, the ECG expanded from Einthoven's 3 leads to the current 12 with the addition of the augmented unipolar limb leads and the unipolar chest leads, mainly as a result of work by Frank Wilson. This was not only crucial for improved diagnosis of myocardial infarction, but clarified the diagnosis of bundle branch block.

In the last few decades, ECG signals have been recorded after digitising the analogue signals, resulting in greater clarity than recording with a hot stylus on heat-sensitive paper. It is now usual to record at least 3 leads simultaneously, as opposed to consecutive strips, which has been helpful in analysing arrhythmias.

\section{Understanding arrhythmias}

While many authors contributed to the understanding of arrhythmias by studying the ECG, the Chicago school of Louis Katz, Alfred Pick and Richard Langendorff was pre-eminent from the 1940s to the 1970s. Leo Schamroth in Johannesburg made huge contributions, not least by the publication of "An Introduction to Electrocardiography" in 1956 and "The Disorders of Cardiac Rhythm" in 1971. During this time, all the major concepts of impulse generation and conduction relevant to arrhythmias were deduced from standard surface ECG recordings.

Laboratory work on isolated hearts and muscle strips delineated cardiac electrical activity at a cellular level and led to an understanding of membrane potentials and ion channels. The understanding of cardiac ion channels received a boost with the elucidation of the genetic defects responsible for the congenital long QT syndromes.

The demonstration by Dirk Durrer and his colleagues in Amsterdam (1967) that tachycardias in patients with WolffParkinson-White (WPW) syndrome could be initiated and terminated with single timed premature stimuli proved the mechanism to be re-entry. ${ }^{(12)} \mathrm{A}$ similar study reported by Hein Wellens and others from the same group indicated that scarrelated ventricular tachycardia was also due to re-entry. ${ }^{(13)}$ These techniques became part of routine clinical EP studies (see below).

\section{Defibrillation and cardioversion}

The concept of electrical termination of ventricular fibrillation (VF) dates back to the 19th century, but the first practical steps toward this goal were taken by Hooker, Kouwenhoven and Langworthy in 1933,(14) Naum Gurvich in Moscow in 1939(15) (who also pioneered DC defibrillation and biphasic waveforms) and Claude Beck in Cleveland in 1947.(16) Beck is credited with the first successful defibrillation of a human being in 1947 , when he resuscitated a young man undergoing surgery using a device that he had designed. Paul Zoll described the first cases of successful external defibrillation. (17) The subsequent development of open heart surgery, cardiac catheterisation, interventional electrophysiology and percutaneous revascularisation 
would be unthinkable without the availability of electrical defibrillation.

Bernard Lown and Barouh Berkovits were responsible for the development of direct current defibrillation and synchronised cardioversion which remain standard to this day, ${ }^{(18)}$ albeit with some modifications to improve the efficiency of the waveform.

\section{Pacemakers}

While a working pacemaker was constructed in the 1930s by Hyman, Paul Zoll is credited with developing the first effective transcutaneous pacemaker to treat Stokes-Adams syndrome due to complete heart block in 1952.(19) Sy Furman, while still a surgical resident at the Montefiore in New York in 1958, developed a transvenous pacing electrode which subsequently became the basis for modern permanent pacing systems. ${ }^{(20)} \mathrm{He}$ remained a leader in the field of cardiac pacing for the rest of his life.

Until the introduction of the implantable pacemaker, heart block was a lethal disease. One year survival was between 30 and 50\%.(21) The first pacemaker was implanted in 1958 into Arne Larsson, a Swedish engineer in his 40s with complete heart block and recurrent syncope. His wife was aware that Elmqvist and Senning at the Karolinska Institute in Stockholm had implanted experimental pacemakers in dogs. She managed to persuade Åke Senning to implant a unit in her husband, who survived another 43 years and many pacemaker replacements. ${ }^{(22)}$ Studies in the 1960s and early 70s, when only simple $\mathrm{VOO}$ and $\mathrm{VVI}$ units were implanted, showed survival rates of $90 \%$ at one year and 5 year survivals of 60 to $80 \%$, despite an average age of around 65 years at the time of implant. ${ }^{(23)}$ Survival was similar to an age-matched cohort from the general population. Very few medical interventions can compare with pacing in respect of dramatic improvements in both quantity and quality of life.

The transistor was critical to the development of implantable pacemakers. Early units were powered by mercury cells which could not be sealed because they emitted hydrogen, resulting in poor reliability. The development of the lithium-iodide battery by Wilson Greatbatch in the early 1970s revolutionised pacemaker design and longevity. ${ }^{(24)}$ Rapid technological advances over the past few decades have resulted in increased sophistication and reliability. Electrodes and leads, however, remain the weakest link which has led to the development of leadless pacemakers. ${ }^{(25)}$

\section{His bundle recording and EP studies}

There had been animal studies in which recordings had been made of His bundle activity, but it was the publication of the catheter technique for recording the His bundle in man, by Benjamin Scherlag and his colleagues in 1969,(26) that opened the way for clinical electrophysiological studies. It was initially widely applied to the study of conduction abnormalities, but soon began to incorporate timed premature atrial and ventricular extrastimuli to induce and terminate tachycardias. Aside from diagnosis of tachyarrhythmias, it was widely used in the 1970s and 80s for testing the efficacy of antiarrhythmic drugs. As catheter ablation of arrhythmias has developed, clinical EP studies have increasingly become therapeutic, rather than purely diagnostic.

Intracardiac recordings, stimulation studies and detailed mapping have validated most of the early concepts of arrhythmias deduced from the ECG and have vastly increased our understanding of clinical arrhythmias.

\section{Antiarrhythmic drugs}

It was recognised early in the 20th century that digitalis preparations could slow the ventricular response in patients with atrial fibrillation. Quinidine was discovered in the 1920s and was found to be useful in converting AF to sinus rhythm and in maintaining that rhythm. Procainamide, lignocaine (lidocaine), disopyramide, mexiletine and many others were added during the 1960s and 70s. Vaughan Williams published a classification of antiarrhythmic drugs in 1970.(27) It was based on their effects on the action potential of isolated muscle strips and correlated with their effects on cardiac ion channels. The drugs, particularly Class I agents, were widely and enthusiastically used both for symptomatic tachyarrhythmias and prophylactically. The latter use arose from observations, made mainly by Bernard Lown, that ventricular fibrillation in patients with acute myocardial infarction was often triggered by premature ventricular complexes (PVCs) falling on the T-wave ("R on T" phenomenon), leading to the assumption that suppression of PVCs by lignocaine would prevent VF and improve survival. Lown's group and others also noted a correlation between the frequency of PVCs in the post-infarction period and the incidence of sudden unexpected death.(28) This lead to the routine use of Class I antiarrhythmics (mainly quinidine in the USA) in survivors of myocardial infarction in whom PVCs were documented by Holter monitoring.

This practice continued until the Cardiac Arrhythmia Suppression Trial (CAST) ${ }^{(29)}$ was undertaken (against much opposition). The trial was stopped prematurely when it became clear that mortality in the active treatment group (using the Class IC agents flecainide, encainide and morizicine) was nearly 3 times that in the placebo group. Review of many smaller trials of Class I agents showed a similar trend.

Quinidine syncope had been noted in the 1920s and again in 1950. The mechanism was shown to be torsades de pointes ventricular tachycardia due to prolongation of ventricular repolarisation. ${ }^{(30)}$ Subsequently, a host of drugs has been shown to prolong the QT interval and promote torsades. The list includes not only Class I and III antiarrhythmics, but large 


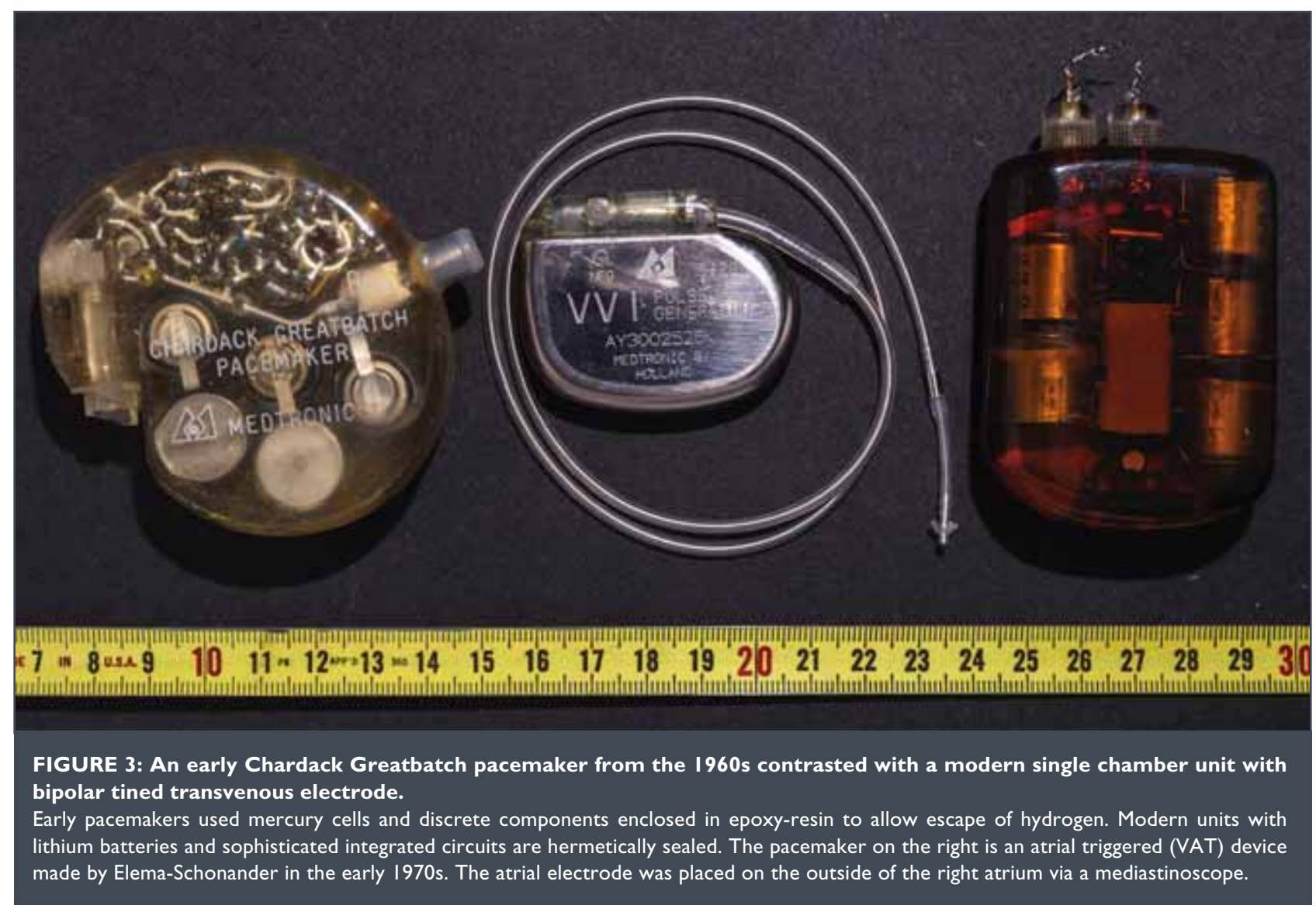

numbers of non-cardiac drugs - antipsychotics, antibiotics, antihistamines, bowel motility preparations, and many others. The list is available at https://www.crediblemeds.org/index.php/ login/dlcheck. This phenomenon of pro-arrhythmia, together with limited efficacy, seriously limits the usefulness of antiarrhythmic drugs.

\section{Surgery}

Recognition of the anatomical substrate for many arrhythmias led to the development of surgical techniques to remove, or ablate, the offending tissue. Nineteen sixty eight saw the first successful surgical division of an accessory AV connection responsible for reciprocating tachycardias in a patient with WPW syndrome.(31) This both proved the concept and provided a cure for the condition. Surgical removal of a left ventricular aneurysm was reported in the 1959 and was shown to prevent recurrent ventricular tachycardia. (32) Subsequent developments in this technique, using intra-operative mapping and selective removal of scar, improved the results considerably.

Surgical modification of the atria to cure AF has been practised since the 1980s. The most successful has been James Cox's maze operation, ${ }^{(33)}$ in which the atria are divided into corridors each too small to sustain the wandering re-entry circuits thought to perpetuate AF. The maze procedure has undergone many modifications and is still used in selected patients at the time of surgery for other conditions, especially mitral valve repair. While around twice as many patients are in sinus rhythm at one year after surgery following mitral surgery and ablation as with mitral surgery alone, no benefit in mortality or stroke has yet been demonstrated. In a recent trial, those having additional AF surgery were significantly more likely to need permanent pacing. ${ }^{(46)}$

\section{LATE 20TH CENTURY}

\section{Catheter ablation}

In 1982, Mel Scheinman and John Gallagher independently published reports of a technique to ablate the AV junction and produce complete heart block by means of a high energy discharge (up to 360J) delivered via the tip of an electrode catheter placed against the His bundle. ${ }^{(34,35)}$ Heart block induced in this manner, together with permanent pacing, was used mainly in patients in AF with ventricular rates uncontrolled by drugs.

Fortunately, in 1987, the safer technique of catheter ablation using radio-frequency (RF) energy was introduced. ${ }^{(36)}$ This has revolutionised the treatment of tachycardias. Together with advances in computerised mapping systems, RF has enabled 
the cure of more than $90 \%$ of patients with WPW syndrome and similar numbers with AV nodal re-entry tachycardia. ${ }^{(37)}$ While procedure-related complications, such as tamponade and heart block still occur occasionally, the short term risks are generally worth it for the long term benefit, compared to the long term risks and poor efficacy of antiarrhythmic drugs.

In 1998, Michel Haissaguerre and his colleagues in Bordeaux observed high frequency discharges originating in the pulmonary veins which appeared to be the triggers for atrial fibrillation. ${ }^{(38)} \mathrm{He}$ was able to ablate these foci with apparent cure of the AF. From this has developed the world-wide explosion in catheter ablation procedures for AF. Results in patients with paroxysmal AF and little or no associated structural heart disease are very good, although more than one procedure is often necessary to achieve the best result. The outcomes in those with long-standing "persistent" AF, particularly with older age and associated heart disease, are not as good. The potential complications and need for repeat procedures make it less attractive. ${ }^{(39)}$

\section{Implantable cardioverter defibrillators}

In 1967, a friend of Michel Mirowski died suddenly. This event spurred Mirowski, a cardiologist in Israel, to develop an implantable cardioverter defibrillator (ICD). He moved to Boston and, together with his colleague Morton Mower and engineers, developed an implantable device capable of automatically delivering a shock of around 30] in response to a heart rate above a pre-set limit. This is sufficient to convert VF via an electrode in direct contact with the heart. The first human implant occurred in 1980.(40) Technological developments over the years have led to the incorporation of sophisticated detection algorithms, overdrive pacing to terminate ventricular tachycardia (VT), transvenous electrodes, smaller size, greater reliability and longer battery life. Trans-venous electrodes, however, are prone to failure and remain the weakest link in the ICD system. Subcutaneous ICDs have now been developed where the ICD lead is positioned outside of the heart, but have the disadvantage of not providing anti-tachycardia pacing of VT or back up bradycardia pacing.

The early units were implanted in patients resuscitated from cardiac arrest or with documented $\mathrm{VT}$ unresponsive to antiarrhythmic drugs. Major trials in the 1990s (MADIT) tested its use prophylactically in patients deemed at high risk for VF those with severely depressed left ventricular function. They showed a modest but definite improvement in survival in comparison to conventional treatment and have become the standard of care in eligible patients. ${ }^{(41)}$

\section{Cardiac resynchronisation therapy (CRT)}

While not a therapy for arrhythmias, the concept and execution uses technology from the field. It has been shown that left bundle branch block (LBBB) with a QRS width of more than 120ms may impair left ventricular function because of asyn- chronous contraction. Correction of this abnormality by simultaneous pacing of the left and right ventricles has been shown to improve LV function in the majority and improves survival in patients with moderate and severe heart failure. ${ }^{(42)}$

\section{EARLY 2IST CENTURY}

Improving technology

The field of cardiac electrophysiology is advancing rapidly with improving technology. It is becoming increasingly popular, particularly with the rapid uptake of ablation for atrial fibrillation. In the USA, in 2010, there was one electrophysiologist for every 127500 people. Most patients with WPW syndrome have had their accessory pathways ablated (the "Wolff' has become an endangered species!). The indications for, and practice of, AF ablation continues to expand. Opportunities for training abound in developed countries and resources allow for relatively unrestricted use of expensive treatments, such as ICDs.
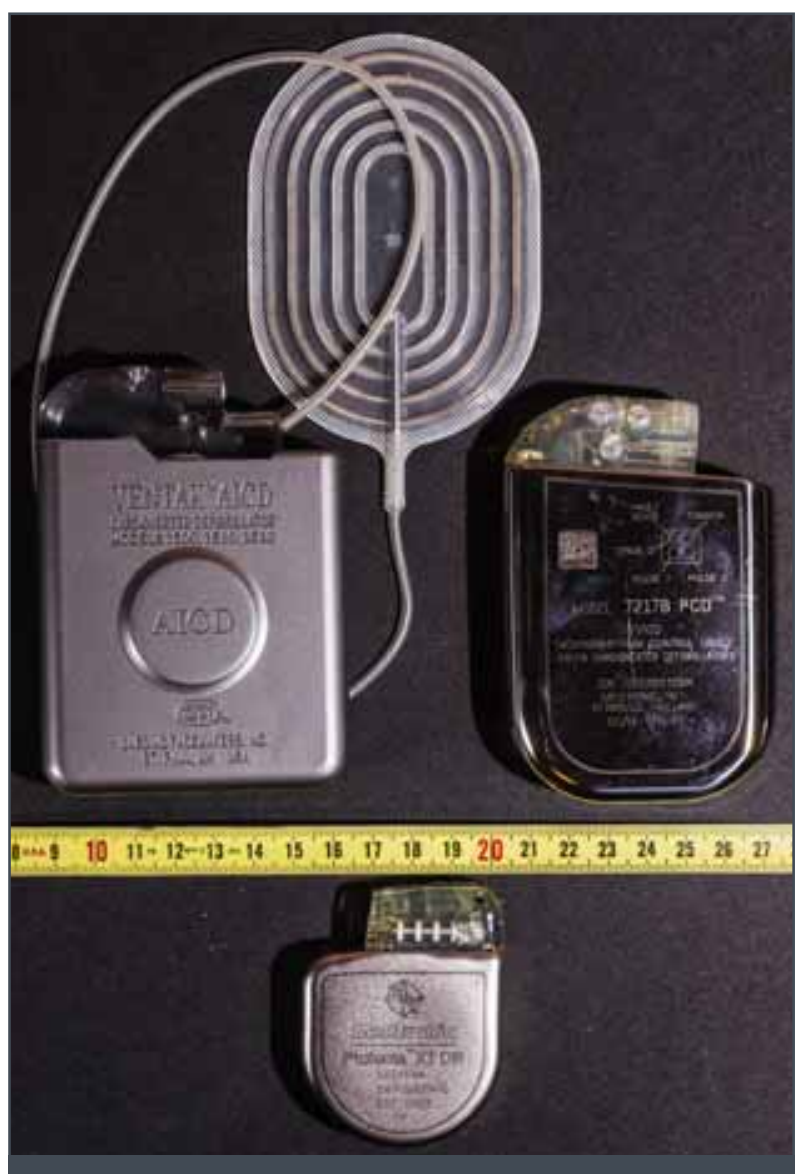

FIGURE 4: Two early ICDs contrasted with a much smaller modern dual chamber ICD (below).

The patch electrode was sutured directly onto the pericardium over the apex, the second electrode being a transvenous coil in the superior vena cava. Modern systems use a single transvenous electrode, the shock being delivered between the electrode and the can of the ICD. 
World usage of pacemakers and ICDs

Every 4 years, Harry Mond of Australia coordinates the World Survey of Cardiac Pacing and Cardioverter-defibrillators, to which South Africa has contributed data since 1972. The most recent published survey was of the calendar year 2009.(43)

In the USA, 767 pacemakers and 434 ICDs were implanted per million of the population in 2009. In Europe, pacemaker implant rates varied from 350 per million (Ireland and Serbia) to 927 in Germany, with most over 500. ICD implants ranged from 14 to 290 per million (Germany), with most over 100.

\section{Pacing and EP in South Africa and Africa}

The picture in South Africa is very different. There are currently only 13 electrophysiologists (EPs) in South Africa accredited by CASSA (Cardiac Arrhythmia Society of South Africa), 12 of whom work predominantly or exclusively in private practice. The ratio of EPs to population is therefore around I for every 4.5 million of the general population. There is, however, only one EP in the public/academic sector in South Africa.

The annual rate of new pacemaker implantations per million of the SA population has risen slowly from around 20 in the 1980s to 60 in 2009, with ICD implants of 6 per million - less than $10 \%$ of that in most developed countries. This figure does not reveal the imbalance in distribution. There are no pacemaker implanters in 4 of South Africa's 9 provinces. In 2005, the implant rate in those with health insurance was about 200/ million, as opposed to less than 10/million for those without. While some of this difference may be due to the different age distribution of the 2 groups, as there is a greater proportion of young people in the non-insured group, most is due to socioeconomic factors and limited access to tertiary health care. Since pacing for heart block is a lifesaving procedure, this imbalance is likely to translate into many deaths which could have been prevented. In addition, access to CRT and ICDs in the public sector is limited by financial constraints and lack of skilled personnel.

The situation in the rest of sub-Saharan Africa is even worse. In a recent 2014 Pan African Society of Cardiology (PASCAR) survey, ${ }^{(44)}$ only 7 other countries implant any pacemakers at all. The number of implants is low and there is a severe shortage of doctors skilled in permanent pacemaker implantation. Most pacemakers that are implanted are funded privately in 1 or 2 specialised centres.

What is to be done? The most pressing needs, in our view, are to improve access to pacing for indigent patients with heart block and to increase the number of pacemaker implanters. While we do not have any evidence that patients who are referred or find their way to a tertiary institution for pacing are denied the procedure, it is likely that many are going undetected at the level of primary health care or are not referred. Distance from a tertiary centre is likely to be a deterrent. Many primary care clinics do not have ECG machines. Efforts to increase awareness of arrhythmias in general and heart block, in particular, among staff in clinics and secondary hospitals need to be undertaken. CASSA has been active in this regard.

We need to get an estimate of the prevalence of conduction disease and serious arrhythmias in the population, particularly among older people. Surveys done more than 40 years ago suggested a lower prevalence among rural elderly blacks than among urban whites of a similar age, ${ }^{(45)}$ but increasing urbanisation may have changed this.

PASCAR has formed a task force to address the problem of heart block and sudden death in Africa. Among other initiatives, it is arranging for health care professionals from other African countries to spend 6 months training in pacemaker implantation and follow up at Groote Schuur Hospital. It is also addressing the issue of pacemaker and ICD re-use.

\section{CONCLUSION}

Cardiac electrophysiology made huge strides in the last century, building on the groundwork done in the 19th century. The invention of the ECG, its development and the continuous refinement of its interpretation has been pivotal in this process.

The implantable cardiac pacemaker was one of the greatest inventions of the 20th century. It converted a debilitating and lethal disease into a condition compatible with a normal productive lifespan.

Catheter ablation, particularly in those with otherwise normal hearts, revolutionised the treatment of tachyarrhythmias. Its scope continues to expand to encompass complex rhythms, such as atrial fibrillation and ventricular tachycardias.

Improving technology contributes to shorter procedures and better outcomes.

Most important, however, is the availability of skilled clinicians and operators to make these advances available to patients. This is particularly true in sub-Saharan Africa. While South Africa is somewhat better served than the rest of the continent, we still fall woefully short of international standards of access. The huge discrepancy in access to lifesaving treatment mirrors the many other inequalities in our society.

\section{ACKNOWLEDGEMENTS (ROB SCOTT MILLAR)}

Pro Obel, mentor and friend, was the pioneer electrophysiologist in South Africa and an early adopter; he continues to be active in the field. The late Andrzej Okreglicki (AO) started as my protégé, but taught me a great deal before his tragic death in 
2012. This article is based on a lecture given in memory of $A O$ at the SA Heart Congress in Durban in 2014.

Conflicts of interest: RSM has received honoraria for teaching from Medtronic, South Africa and AC has received lecture fees/honoraria from Medtronic and St Jude Medical.

\section{REFERENCES}

I. Fye WB. The origin of the heart beat: A tale of frogs, jellyfish, and turtles. Circulation 1987;76:493-500

2. Silverman ME, Grove D, Upshaw CB Jr. Why Does the Heart Beat? The Discovery of the electrical system of the heart. Circulation. 2006; I 13: 2775-2781.

3. Silverman ME, Upshaw CB Jr. Walter Gaskell and the understanding of atrioventricular conduction and block. J Am Coll Cardiol 2002;39:1574-80.

4. Suma K, Sunao Tawara. A father of modern cardiology. PACE 200 I;24:88-96.

5. Silverman ME, Hollman A. Discovery of the sinus node by Keith and Flack: On the centennial of their 1907 publication. Heart 2007;93; I I84- I 187.

6. Upshaw CB Jr, Silverman ME. Luigi Luciani and the earliest graphic demonstration of Wenckebach periodicity. Circulation. 2000; 102:2662-2668.

7. Burchell HB. A centennial note on Waller and the first human electrocardiogram. Am J Cardiol 1987;59:979-983.

8. Einthoven $\mathrm{W}$. The string galvanometer and the measurement of the action currents of the heart. Nobel Lecture, December, 1925

9. Craib WH. A study of the electrical field surrounding active heart muscle. Heart 1927;14:7|-109.

10. Silverman ME. From rebellious palpitations to the discovery of auricular fibrillation: Contributions of Mackenzie, Lewis and Einthoven. Am J Cardiol 1994;73:384-389

1।. Mines GR. On dynamic equilibrium in the heart. J Physiol 19/3;46;349.

12. Durrer D, Schoo L, Schuilenburg RM, Wellens HJJ. The role of premature beats in the initiation and the termination of supraventricular tachycardia in the Wolf-Parkinson-White Syndrome. Circulation 1967;36:644-662.

13. Wellens HJJ, Schuilenburg RM, Durrer D. Electrical Stimulation of the heart in patients with ventricular tachycardia. Circulation 1972;51:216-226.

14. Hooker DR, Kouwenhoven WB, Langworthy OR. The effect of alternating electrical currents on the heart. Am J Physiol 1933;103;444.

15. Efimov IR. Naum Lazarevich Gurvich (1905 - 1981) and his contribution to the history of defibrillation. Cardiology J 2009;16:190-193.

16. Beck CS, Pritchard WH, Feil HS. Ventricular fibrillation of long duration abolished by electric shock. JAMA 1947; 135:985-986.

17. Zoll PM, Linenthal AJ, Gibson W, et al. Termination of ventricular fibrillation in man by externally applied electric countershock. N Engl J Med 1956; 254:727-732.

18. Lown B, Neuman J, Amarasingham R, Berkovits BV. Comparison of alternating current with direct current electroshock across the closed chest. Am J Cardiol 1962; 10:223-233.

19. Zoll P. Resuscitation of the heart in ventricular standstill by external electric stimulation. N Engl J Med 1952;247:768-77।.

20. Furman S, Robinson G. The use of an intracardiac pacemaker in the correction of total heart block. Surg Forum 1958;8:245-248.

21. Friedberg CK, Donoso E, Stein WG (1964). Nonsurgical acquired heart block. Annals of the New York Academy of Sciences, III, 835.

22. Larsson B, Elmqvist $H$, Ryden $L$, Schuller $H$. Lessons from the first patient with an implanted pacemaker 1958 - 200 I. PACE 2003:26: I | 4- I 24.

23. Zion MM, Marchand PE, Obel IWP. Long-term prognosis after cardiac pacing in atrioventricular block. Br Heart J 1973;35:359

24. Furman S. The early history of cardiac pacing. PACE 2003;26:2023-2032.

25. Reddy VY, et al. Permanent leadless cardiac pacing: Results of the LEADLESS trial. Circulation. 2014;129:1466-|47|.

26. Scherlag $B$, et al. Catheter technique for recording His bundle activity in man Circulation 1969;39:13-18
27. Vaughan Williams EM. Classification of anti-arrhythmic drugs. In symposium on cardiac arrhythmias, Edited by $\mathrm{E}$ Sandoe, E Flensted-Jensen and $\mathrm{KH}$ Olesen. Sweden, AB ASTRA, Sodertalje 1970:449-4721.

28. Lown B, Wolf M. Approaches to sudden death in coronary heart disease. Circulation 1971;44:130-142.

29. The Cardiac Arrhythmia Suppression Trial (CAST) investigators. Preliminary report: Effect of encainide and flecainide on mortality in a randomised trial of arrhythmia suppression after myocardial infarction. N Engl J Med 1989;321:406-412.

30. Selzer A, Wray HW. Quinidine Syncope: Paroxysmal ventricular fibrillation occurring during treatment of chronic atrial arrhythmias. Circulation. 1964;30:17-26.

31. Cobb FR, Blumenschein SD, Sealy WC, et al. Successful surgical interruption of the bundle of Kent in a patient with Wolff-Parkinson-White Syndrome. Circulation. 1968;38:1018-1029.

32. Couch OA Jr. Cardiac aneurysm with ventricular tachycardia and subsequent excision of the aneurysm - case report. Circulation 1959;20:251-253.

33. Cox JL, Schuessler RB, Boineau JP. The development of the maze procedure for the treatment of atrial fibrillation. Seminars in Thoracic and Cardiovascular Surgery 2000; 12:2-14.

34. Gallagher JJ, Svenson RH, Kasell JH, et al. Catheter technique for closed-chest ablation of the atrioventricular conduction system: A therapeutic alternative for the treatment of refractory supraventricular tachycardia. N Engl J Med 1982;306:194-200.

35. Scheinman MM, Morady F, Hess DS, Gonzalez R. Catheter-induced ablation of the atrioventricular junction to control refractory supraventricular arrhythmias. JAMA 1982;248:85I-855.

36. Huang SK, Bharati S, Graham AR, et al. Closed chest catheter desiccation of the atrioventricular junction using radiofrequency energy: A new method of catheter ablation. Am Coll Cardiol 1987;9:349-58.

37. Calkins H, Yong P, Miller JM, Olshansky B, et al. for the Atakr Multicenter Investigators Group. Catheter ablation of accessory pathways, atrioventricular nodal reentrant tachycardia, and the atrioventricular junction: Final results of a prospective, Multicenter clinical trial. Circulation 1999;99:262-270.

38. Haïssaguerre M, Jaïs $P$, Shah D, Takahshi A, et al. Spontaneous initiation of atrial fibrillation by ectopic beats originating in the pulmonary veins. $N$ Engl J Med 1998;339:659-666.

39. Calkins $\mathrm{H}$. Catheter ablation to maintain sinus rhythm. Circulation. 2012; |25:|439-| 445.

40. Mirowski M, Reid PR, Mower MM, et al. Termination of malignant ventricular arrhythmias with an implanted automatic defibrillator in human beings. N Engl J Med 1980;303;322.

4I. Moss AJ, Hall J, Cannom DS, Daubert JP, et al. For the Multicenter automatic defibrillator implantation trial investigators. Improved survival with an implanted defibrillator in patients with coronary disease at high risk for ventricular arrhythmia. N Engl J Med 1996;335:1933-40.

42. Moss AJ. Preventing Heart failure and improving survival. N Engl J Med 2010;363:2456-7.

43. Mond HG, Proclemer A. The IIth world survey of cardiac pacing and implantable cardioverter-defibrillators: Calendar year 2009 - A world society of arrhythmia's project. PACE 201 1;34:1013-1027.

44. Chin A. Managing arrhythmias in Africa. Presented at Cardiostim meeting, Nice, France June 2014.

45. Obel IWP, Scott Millar RN, Lewis CM, et al. The incidence of conduction disturbances in the elderly in the Republic of South Africa and their treatment by pacing in 1972. In cardiac pacing: Proceedings of the IVth International Symposium on Cardiac Pacing, Groningen, the Netherlands, April 17-19, 1973. Ed. Thalen H J Th. 1973, Van Gorcum, Assen - the Netherlands.

46. Gillinov AM, et al. Surgical ablation of atrial fibrillation during mitral-valve surgery. N Engl J Med 20 15;372:1 399-409. 